Horizons philosophiques

\title{
Lassitude et acharnement
}

\section{Promenade existentielle chez Beckett et Deleuze}

\section{Vincent Jacques}

Volume 17, numéro 1, automne 2006

Existentialisme et philosophie continentale

URI : https://id.erudit.org/iderudit/802964ar

DOI : https://doi.org/10.7202/802964ar

Aller au sommaire du numéro

Éditeur(s)

Collège Édouard-Montpetit

\section{ISSN}

1181-9227 (imprimé)

1920-2954 (numérique)

Découvrir la revue

\section{Citer cet article}

Jacques, V. (2006). Lassitude et acharnement : promenade existentielle chez Beckett et Deleuze. Horizons philosophiques, 17(1), 13-30.

https://doi.org/10.7202/802964ar d'utilisation que vous pouvez consulter en ligne.

https://apropos.erudit.org/fr/usagers/politique-dutilisation/ 


\title{
Lassitude et acharnement Promenade existentielle chez Beckett et Deleuze
}

\author{
"Le besoin de parler, ce besoin de s'arrêter, cette impossi- \\ bilité de s'arrêter" \\ Beckett
}

Il fut, il est et sera toujours des systèmes philosophiques projetant une éthérée architecture du monde à la place de l'énigme que pose celui-ci. Énigme aussi d'être au sein de celui-ci. Réconfort de la stabilité idéelle, logique, mathématique etc., mise à mal, éventée par le cri du penseur solitaire, posant son existence contre le système. "Tout ne va pas de soi" ou "Tout ne devrait pas aller de soi», tel est d'après nous l'une des exigences fondamentales de l'existentialisme aujourd'hui, tout comme elle l'était hier, exigence tirée du fond de l'inconfort de l'existant, plus que nécessaire dans ce monde moderne où confort et facilité sont roi et reine. L'insistance donnée au cri de l'existant, nous la poursuivrons chez Beckett et Deleuze, deux auteurs souterrainement, c'est-à-dire sans références explicites (ou si peu), travaillés et influencés par ce courant de pensée qu'on a appelé l'existentialisme. Attention, par contre, en ce qui a trait à Beckett, ne pas lui affubler de force les traditionnelles inepties sur l'absurdité et l'incommunicabilité, thèmes qui sentent un peu trop le brouet théologique.

Nous développerons plutôt trois grands thèmes que sont Autrui, la Bêtise et l'Innommable', et ce aussi bien chez Beckett que chez Deleuze. On pourrait dire qu'à travers Autrui une visée critique se déploie, que la bêtise est l'une des armes de cette critique (mauvaise volonté chez Deleuze) et que l'innommable, finalement, est ce sur quoi l'existant, une fois dissolue la forme sujet, devrait déboucher : devenir-imperceptible, se couler dans le monde pour Deleuze, exil et acharnement entre la vie et la mort, entre les mots et le silence pour Beckett - se déprendre du monde. Entrelacée à ces thèmes, nous essaierons aussi de penser une posture existentielle, la lassitude, et son corollaire, cette petite intranquillité au fond de l'existant que nous appellerons l'acharnement, source du cri propre à l'existant homme, passion d'errance. 
Nous essaierons ainsi de répondre à la question : qu'en est-il de la philosophie de l'existant aujourd'hui?, une fois le "romantisme» et certaines grandes idées de l'existentialisme de naguère dissipés : vérité, authenticité, être soi, être vrai. Selon nous Deleuze et Beckett, chacun à leur manière, recueillent l'héritage d'une philosophie centrée sur l'existant pour le faire fructifier de manière essentielle à sa survie, peut-être l'existentialisme sans un certain 'héroïsme' qui faisait hier encore sa fortune. (Nous montrerons aussi plus particulièrement que Beckett n'est pas cet auteur nihiliste que l'on décrit trop souvent et que Deleuze n'est pas cet anarchiste désirant, jovial et optimiste à outrance.) II s'agira d'exposer le radicalisme critique des conceptions de nos deux auteurs, ce que nous ferons dans les deux premières parties de notre exposé, sur Autrui et sur la bêtise (mauvaise volonté), radicalisme qui met à mal le sujet, support de ces notions existentialistes traditionnelles que sont l'authenticité et la responsabilité. Lassitude. Aller au-delà de l'habitude d'être Je. On montrera finalement que cette entreprise de démolition n'est pas pure passion nihiliste, mais effort pour entendre, laisser place à un étrange et problématique rapport au monde, passion de l'existant une fois le sujet dissous, rapport au monde problématique, indicible, innommable propre à l'existant homme, acharnement d'un petit quelque chose propre à l'homme, constitutif de son inquiétude perpétuelle. Posons ici l'hypothèse que l'existentialisme devait passer outre le sujet et ses petits et grands tourments, pour atteindre enfin une inquiétude plus fondamentale?2.

\title{
Autrui, viscosité d'une jactance abhorrée et structure toute-puissante
}

\author{
"L'air est plein de nos cris. Mais l'habitude est une grande \\ sourdine"
}

Beckett, En attendant Godot, p.128

L'habitude. II s'agit pour Beckett de s'en extirper, de s'extraire de la jacasserie permanente de la tribu. "La vérité est le pet du plus grand nombre»3. Pas de valeur autre que numéraire, donc, à toutes ces vérités qui sont charriées, qu'on le veuille ou non, à travers nous. Car, comme le dit si bien Lacan, l'homme n'est pas tant un être parlant qu'un être parlé. Celui qui se croit sujet (parlant) est en fait parlé, c'est la tribu qui parle à travers lui, tout le flux de langage qui le traverse vient d'Autrui. Le langage est la grande sarabande d'Autrui, ou comme le disait Sartre, l'existence en présence d'Autrui. «Mais 
c'étaient surtout mes semblables qu'ils voulaient me faire avaler»4. Pour Beckett, Autrui, via le langage, aliène l'existant, logorrhée frénétique de ratiocinations, de preuves, de justifications, de constats, d'évidences et de clichés qui constituent cette belle et rassurante totalité de l'habitude. II y aura donc haine de l'habitude, répétition et reproduction des manies d'Autrui. Pourquoi? Parce que ces manies d'Autrui couvrent quelque chose, assourdissent de leur inlassable babil ce murmure, cette inlassable morsure du cri dès lors inaudible à la conscience, entité donnée, problématique, qu'il ne faut surtout pas confondre avec le sujet, avatar d'Autrui lui aussi.

Car pour que le système de l'habitude marche à plein régime, pour qu'un sujet ait ses habitudes, il faut qu'il soit constitué comme sujet pour lui - par Autrui, habitude d'être sujet, renvoi pour soi par Autrui d'une identité fixe, habitude, habituelle : "Mais voyons, mon cher, voilà, voilà qui vous êtes, regardez cette photo, et voici la fiche, pas de condamnations, je vous assure, faites un effort, à votre âge, être sans identité, c'est une honte, je vous assure" 5 . Je corresponds au rôle, à ce que l'on attend de moi, mauvaise foi sartrienne, je suis tel qu'Autrui me perçoit, tel que son dire me traverse, me constitue et me force à dire, à m'avouer comme sujet. À cette entité qui croit dire et se dire, tandis qu'elle est dite, des habitudes de dire s'attribuent, confort mollasson de l'ensemble grégaire des existants. De ce confort et de cette mollesse, Beckett est profondément las, lassitude comme mélange d'ennui et de dégoût pour toutes ces habitudes, cloaque visqueux d'Autrui. Lassitude donc, pour un ordre des choses qui trop facilement va de soi, qui ne laisse pas place à la petite intranquillité existentielle enfouie au sein de l'existant-homme : "De sorte que, s'il n'y avait pas l'habitude, la vie devrait paraître délicieuse à des êtres qui seraient à chaque heure menacés de mourir - c'est-à-dire à tous les hommes"6. Dans son essai sur Proust, Beckett accuse l'habitude d'étouffer la "souffrance d'être», c'est-à-dire "le libre jeu de toutes nos facultés" ou "état provisoire de lucidité aiguë 7". L'habitude paralyse et anesthésie les facultés qui n'ont pas une importance strictement vitale et utilitaire. Un tout petit nombre alors de facultés échappent à la somnolence pour effectuer, via l'habitude, leur utilitaire besogne. L'homme est ainsi balancé entre les deux pôles que sont l'ennui et la souffrance, ennui de la simple reproduction sans remous de la vie et souffrance d'exister. Beckett choisira résolument le deuxième pôle, que tant d'autres pourtant s'efforcent de fuir, d'étouffer, d'anesthésier grâce à l'habitude et à l'action. 
Le monde d'Autrui c'est ainsi le monde frénétique de l'habitude et de l'action, du faire et du dire qui renvoient toujours plus vite à d'autres faires et d'autres dires, frémissement et vacarme continus que peuvent supporter les sujets, ils sont même faits pour ça, mais qui annihilent la conscience, la vraie, sans sujet, celle que cherche Beckett, celle qui admet, sait entendre l'énigmatique cri de la souffrance. Fuir cette aliénation d'Autrui exige donc une démolition en règle du sujet et un effort constant pour se déprendre du monde qui, selon Beckett, ne peut se déployer qu'à travers le déjà-dit et le déjàressassé propre au sempiternel babil de la tribu. Pour faire place au cri et atténuer l'effet de sourdine, il faudra aux personnages beckettiens la solitude, l'errance du solipsisme et l'immobilité, voire l'inconfort physique. Une remarque ici, sur un certain radicalisme langagier chez Beckett, où plutôt sur l'enfermement que le langage nous impose. Prenons par exemple le raisonnement de Malone sur son bâton. Au diable, l'idée du bâton dit-il, vapeur idéaliste inutile, l'important est ce bâton-là, mon bâton, ce bâton-ci, celui qui, bâtonnant à ce moment ou à un autre, pragmatise, trace à même le réel..., mais le bâton, même ce bâton-ci, il est parlé, dans son être de l'homme il parle, l'existant conscient pense avoir, c'est-à-dire pense pouvoir mouvoir le bâton, mais la conscience n'est qu'agie par la parole qui s'acharne à parler bâton, pas le fumeux universel, mais ce bâton-ci, n'empêche que tous les ci du monde n'ont jamais mû de bâtons... Étrange spinozisme de Beckett où dans un parallélisme corpsmonde/pensée-mots, la première série est un insolite donné pour la conscience qui ne l'entend que par la deuxième série conçue comme cacophonie d'Autrui qu'il faudra démolir. Ou plus exactement doit-on parler ici de l'influence décisive de Geulincx dans l'œuvre de Beckett : "Je suis seulement spectateur de cette machinerie" (sum nudus spectator hujus machinae) ${ }^{8}$. Chez le philosophe belge, une Inspectio sui débouche sur la conscience d'une variété infinie de pensées dans le moi et sur la découverte d'une problématique unité en tant que je, et ce parce que cette conscience reconnaît qu'elle n'est pas la cause de ses pensées. Le je sera dès lors réduit au rôle de spectateur d'une machine où enchaînement de pensées et enchaînement des actes se produisent par le seul élan d'un Dieu Moteur-Acteur-Auteur. Plus de Dieu chez Beckett, mais toujours cette conscience énigmatique qui est le jouet de puissances dont elle n'a pas l'intelligence, conscience spectatrice qui est parole se questionnant elle-même comme parole. C'est dans et par les mots que l'existant est prisonnier, aliéné par Autrui, série qu'il faudra limer, laminer : trouer le langage. Pour 
échapper autant que possible à Autrui, il faudra éliminer le monde. Comme le propose Gauer ${ }^{9}$, on peut dire ici que Beckett prend le contre-pied de la position sartrienne et de sa démarche centrifuge, de l'existant au monde, pour adopter une démarche centripète, du monde à la conscience, du monde encore présent dans les premières œuvres et qui tendra à disparaître au profit du pur non-lieu de la conscience et des voix, des mots qui y résonnent.

Une fois la lassitude ayant mis à mal l'évidence et la cohérence des raisonnements d'Autrui, il reste une fascination pour les mots et leurs acharnements, malgré tout, à se dire : «l'expression du fait qu'il n'y a rien à exprimer, rien avec quoi exprimer, rien à partir de quoi exprimer, aucun pouvoir d'exprimer, et tout à la fois, l'obligation d'exprimer»10. Acharnement de l'expression, quelque chose s'acharne, ça continue, ça ne peut que continuer... L'homme est parlé, acharnement de ce parlé. Dans la conscience et sa souffrance d'être il y aura toujours et toujours quelques restes et scories d'Autrui : tant qu'il y aura des mots...

Deleuze aussi pense Autrui comme chape occultant quelque chose. Sa conception d'Autrui, développée à partir de celle de Tournier, est exposée dans deux courts passages de Différence et répétition ainsi que dans l'article sur Vendredi et les limbes du pacifique repris dans Logique du sens. II y aura pour commencer un hommage à la conception sartrienne d'Autrui comme allant au-delà de l'alternative autrui-objet ou autrui-sujet en concevant Autrui comme "structure propre ou spécificité irréductible à l'objet et au sujet»11. Mais, nous dit Deleuze, Sartre retombe dans l'alternative sujet/objet en conditionnant la structure comme regard: on me constitue objet lorsqu'on me regarde, je suis sujet quand je regarde autrui dès lors objet. «Mais Autrui n'est ni un objet dans le champ de ma perception, ni un sujet qui me perçoit : c'est d'abord une structure du champ perceptif, sans laquelle ce champ dans son ensemble ne fonctionnerait pas comme il le fait»12. II ne faut donc pas confondre Autrui a priori et cet autrui-ci, cet autrui-là. Autrui a priori comme structure se comprend en tant que charpente du possible, qui n'est pas quelque chose d'inexistant, mais plutôt quelque chose enveloppé dans Autrui comme expression d'un monde possible que je pourrai expliquer en l'exprimant à mon tour, c'est-à-dire en l'actualisant. Le possible qui s'offre à moi est toujours enveloppé dans Autrui. Mes petites et grandes frayeurs sont l'expression-développement des possibles enveloppés dans Autrui, structure qui me coupe de la 
grande étrangeté ou de la grande fureur de la nature et de ses éléments.

"Le découpage d'objets, les transitions et les ruptures, le passage d'un objet à un autre, et même qu'un monde passe au profit d'un autre, le fait qu'il y ait toujours quelque chose d'impliqué qui reste encore à expliquer, à développer, tout cela n'est rendu possible que par la structure-Autrui et son pouvoir expressif dans la perception»13. Le fond, la forme, la profondeur, les objets, le temps comme distribution d'une chose qui passe et d'une autre qui la remplace, toutes ces déterminations du monde existent grâce à la structure Autrui peuplant "le monde d'une rumeur bienveillante». Le monde est mon salon. Quel ennui. «Même le désir, qu'il soit désir d'objet ou désir d'autrui dépend de la structure»14. Tout cela est fort ennuyant, nous dira Deleuze, parce qu'ainsi nous sommes condamnés à vivre dans le monde de la représentation à qui l'on reproche : "d'en rester à la forme d'identité, sous le double rapport de la chose vue et du sujet voyant»15. Affres de l'utilitaire. Autrui et le système de la représentation m'aliènent le monde en le réglant, le mesurant, le rabotant pour m'en donner une pâle copie. Grâce au lit de Procuste de la représentation le monde est pour moi un domaine pré-balisé où se déploient mes perceptions, mes peines et mes envies. L'homme habite l'homme. Lassitude deleuzienne, donc, envers ce monde constitué par Autrui a priori, kyrielle de petits blocages forgeant, imposant le système monde-sujet-objet-évidence au détriment du mouvement intense et souterrain de la vie, fureur intense des éléments. "En l'absence d'Autrui, la conscience et son objet ne font plus qu'un»16. Ce que vise Deleuze c'est la rencontre, non pas amour du prochain, mais contact avec l'intensité comme chair de l'Être, recherche du différent ou de l'intensif dans la pensée comme dans toute chose. Le travail critique et créatif de Deleuze sera donc un effort constant en vue de passer outre la structure Autrui et son corollaire qu'est le système de la représentation, et ce pour pointer vers un énigmatique devenir-imperceptible. Nous y reviendrons.

Une autre forme d'aliénation, toujours causée pas Autrui, est développée dans le domaine plus strictement circonscrit du langage. Dans Mille Plateaux, Deleuze et Guattari définissent le langage comme transmission de mots d'ordre et non comme communication d'une information. Ainsi, la maîtresse d'école, quand elle enseigne une règle, n'informe pas l'élève, mais transmet des mots d'ordre : «les commandements du professeur ne sont pas extérieurs à ce qu'il nous 
apprend, et ne s'y ajoutent pas. Ils ne découlent pas de significations premières, ils ne sont pas la conséquence d'informations : l'ordre porte toujours et déjà sur des ordres, c'est pourquoi l'ordre est redondance»17. L'énoncé est l'unité élémentaire du langage, énoncé conçu comme mot d'ordre. Si le langage a pour fonction le mot d'ordre, c'est qu'il est discours indirect. En effet, le langage ne se définit pas comme passerelle entre quelque chose vu et quelque chose dit, mais sautille toujours d'un dire à l'autre. Le langage n'est pas calque, c'est-à-dire témoignage d'une vision, mais ne peut être que la transmission de ce que l'on a entendu dire. La communication, donc, n'est que seconde et restreinte au sein de cette entité langagière première qu'est le discours indirect et dont la fonction essentielle est la transmission. Si cette transmission est mot d'ordre, qu'il ne faut pas confondre avec les commandements explicites, genre particulier et restreint de mots d'ordre, c'est que le langage est ici considéré comme une pragmatique. Austin, en effet, découvre qu'il n'existe pas seulement des rapports extrinsèques entre l'action et la parole, mais qu'il y a aussi plus fondamentalement des rapports intrinsèques entre la parole et l'action qui se déploie dans le dire. Les actes de parole sont une fonction première du langage. Est donc mot d'ordre : "le rapport de tout mot ou tout énoncé avec des présupposés implicites, c'est-à-dire avec des actes de parole qui s'accomplissent dans l'énoncé, et ne peuvent s'accomplir qu'en lui. Les mots d'ordre renvoient donc à tous les actes qui sont liés à des énoncés par une "obligation sociale". II n'y a pas d'énoncé qui ne présente ce lien, directement ou indirectement»18. Bref, comme le disait Sartre, le langage c'est l'existence en présence d'Autrui, c'est un enchaînement "d'obligations sociales» qui m'enchaînent et m'aliènent, dont je suis la victime mais aussi le complice, tant que je participe à la transmission, tant que je prends part au grand tohu-bohu de la jactance tribale.

\section{Bêtise, ignorance et mauvaise volonté}

«Mais c'étaient surtout mes semblables qu'ils voulaient me faire avaler. Ils y mettaient un zèle et un acharnement incroyable»19. Alors ne plus participer à la commune jactance. Ne plus vouloir avaler. Mais c'est qu'ils y tiennent et bien malin celui qui peut résister à Autrui, si, toutefois, cela est bien possible. Car tout va tellement de soi, tout coule de source, et le plus terrible est que tout s'enchaîne automatiquement dans ce brouhaha de l'assourdissante "sourdine de l'habitude" avec tous ses bien sûr, par contre, voyez-vous, 
évidemment... Alors que faire? Les personnages de Beckett utiliserons les armes de la bêtise et de l'ignorance : "et pour ce qui est de laisser de côté l'essentiel, je m'y connais je crois...". La bêtise est cette manière de ne pas enchaîner selon les obligations sociales et l'habitude et l'ignorance le refus de ne pas avaler ce qu'il faut savoir. Grâce à la bêtise on s'acharne à ne pas comprendre, on bloque l'évidence de l'écoulement ratiocinateur. Ne passera pas à travers moi ce qui passe trop facilement à travers tous les autres. On dit de celui qui est bête qu'il est buté. Par ailleurs, pour le sujet, Autrui exige aussi un but, un arrêt, des plages de stabilité bien délimitées. Alors, là, ne plus s'arrêter : "Je n'en suis nulle part, c'est ça qui les travaille, ils veulent que j'en sois quelque part»20. Ne plus s'arrêter, quand la «moralité d'État civil» (Foucault) ou celle, diffuse, du développement personnel le demande - où en êtes-vous? Se couler dans la bêtise c'est donc en un certain sens inverser les rapports si chers à la tribu : s'arrêter quand il faut transmettre, reproduire et poursuivre, ne plus s'arrêter quand une toute simple fixité ou délimitation est demandée.

Du moins à la limite, on assimilera la bêtise à une déficience pathologique : ce n'est pas de sa faute, il est bête. Mais, par contre, l'ignorance, comme comportement sciemment revendiqué, est un affront à la sacro-sainte volonté de savoir, pure et désintéressée, et à son corollaire la curiosité comme fidèle servante et vertu. "Et toutes ces questions que je me pose. Ce n'est pas dans un esprit de curiosité»21. Car, nous dit Beckett, même dans sa forme la plus désincarnée, la curiosité exhale un parfum un peu trop entêtant d'utilité. "La curiosité, ce sont les poils hérissés de notre habitude»22, désir toujours renouvelé de confort, d'abandon à du déjà-ressassé, recherche effrénée de ce doux anesthésiant qu'est la chaleur d'Autrui. La curiosité intellectuelle est trop souvent un moyen de s'en remettre à Autrui en toute bonne conscience, et la lecture, comme l'affirmait Schopenhauer, n'est pas toujours une panacée : «Quand nous lisons, un autre pense en nous, nous répétons simplement son processus mental»23. L'ignorance, qui du dehors est un affront à la moralité de la volonté de savoir inhérente à tout individu sainement constitué, en réalité simple obligation sociale de retrouver pour soi, pour le sujet, des vérités sociales, du déjà-dit, et ainsi assumer ces vérités en tant que sujet (volonté de savoir qui ne doit pas par contre se prendre elle-même comme objet), l'ignorance, donc, est surtout, d'un point de vue existentiel, une manière radicale de ne pas assumer, de ne pas avaler, les discours, les pensées d'Autrui. 
Intolérance au trop plein de corps étrangers, les mots d'Autrui. L'ignorance, donc, est un moyen de ne pas aggraver la situation. Alors, comme conséquence de cette intolérance, refus de l'escroquerie du dialogue comme supposé rapport entre deux sujets constitués s'enrichissant mutuellement. Comment en effet échanger ce que l'on n'a pas? Échanger n'est qu'un moyen de mieux m'aliéner en me fixant comme sujet, qui du coup peut être rempli de tous les mots et raisonnements d'Autrui, et me permet du coup de mieux fixer d'autres sujets qui du coup... Le dialogue n'est nullement un moyen de mieux savoir, de mieux penser, mais au contraire une façon de mieux m'aliéner en m'attachant plus solidement à Autrui. "Je me mis enfin à réfléchir, c'est-à-dire à écouter plus fort” (Molloy). Le discours étant fait de mots, l'enchaînement du discours doit être brisé, le langage doit être troué, pour que la conscience puisse écouter plus fort, pour qu'elle puisse faire résonner les mots. Le dialogue ne fait que rajouter à la sourdine de l'habitude entravant toute écoute. Une fois brisée l'évidence des discours résonnent des "voix". Remarquons ici la similarité avec la conception du langage selon Heidegger24, à cette petite différence près, elle est énorme, que les voix charriées par le langage ne sont pas celles de l'être, mais celles des mots morts.

À un critique qui lui demandait le sens de ses pièces Beckett répondit : "Le mot-clé des mes pièces est peut-être»25. Peut-être est cette volonté des personnages de ne pas savoir, leur acharnement à ne pas comprendre. Peut-être est aussi cette façon de ne pas s'engager dans une piste du monde excluant les autres possibles, trop simple chemin de l'habitude et de l'action. Peut-être est une manière de remettre en question l'ordre des choses, l'ordre du discours en le faisant éclater dans une combinatoire de possibles. Tout le problème de l'œuvre de Beckett sera alors d'épuiser le possible, pour vraiment se déprendre du monde et de la viscosité d'Autrui. Entre temps, mieux vaut être bête et clamer son ignorance que trop se compromettre.

Tout le monde sait, personne ne peut nier. II y a une force tranquille de l'évidence nous dit Deleuze et le "tout le monde sait..." est la forme de la représentation par excellence. Toute pensée se complet dans les présupposés, même la philosophie qui prétend commencer sans eux. C'est que Deleuze appelle "l'Image de la pensée": le soubassement non-questionné sur lequel se bâtit raisonnements et discours. Une de ses plus solides assises est le présupposé d'une pensée naturelle commune à tous. Celle-ci est 
naturellement droite et il suffit donc simplement de la bonne volonté du penseur pour en récolter les fruits. Présupposés subjectifs à la Descartes qui ont leurs racines dans une image morale de la pensée, seul le bien pouvant en effet fonder l'affinité du vrai et de la pensée. Plus simplement, il existe aussi la multitude de présupposés inhérents à toute société. Comment donc échapper à la bonne volonté sociale et subjective? Dans la douce houle du sens commun surgissent parfois des cris isolés et passionnés qui nient que l'évidence coule de source. "Un homme du sous-sol, qui ne se reconnaît pas plus dans les présupposés subjectifs d'une pensée naturelle que dans les présupposés objectifs d'une culture du temps (...). II est l'Intempestif, ni temporel, ni éternel. Ah, Chestov, et les questions qu'il sait poser, la mauvaise volonté qu'il sait montrer»26.

Contre l'écoulement tranquille des présupposés, une arme de choix est la mauvaise volonté, non pas esprit chicaneur qui oppose un raisonnement à un autre, mais refus radical des prémisses mêmes, des soubassements propres aux raisonnements. Ce qui sape toute possibilité de discussion. L'homme de mauvaise volonté met soudainement certains soubassements de l'écoulement du discours en lumière et se retire du fait même du concert des échanges intersubjectifs. Et dire que l'une des dernières marottes de la philosophie contemporaine est justement la communication : «la philosophie ne trouve aucun refuge ultime dans la communication qui ne travaille en puissance que des opinions pour créer du 'consensus' et non du concept»27. C'est le serpent qui se mord la queue : partir de présupposés pour recréer de nouveau du consensus. Mais plus profondément il y a chez Deleuze un refus du dialogue soi-disant constructif, un déni du supposé enrichissement que procurerait la discussion : «'art de construire un problème, c'est très important : on invente un problème, une position de problème, avant de trouver une solution. Rien de tout cela ne se fait dans une interview, dans une conversation, dans une discussion (...) les objections c'est encore pire (...) les objections n'ont jamais rien apporté»28.

La véritable trouée du consensus est en effet celle du problème. Le problème est un cri, un plaie béante dans le monde confortable du «tout le monde sait...» qui jamais ne peut être refermée par les solutions qui s'engouffrent dans sa béance pour la colmater. Pour Deleuze l'essence de la pensée est le problématique, faculté de créer des problèmes qui s'opposent à la bêtise comme faculté de créer de 
faux problèmes. Les faux problèmes sont des poses temporaires décalquées sur les solutions, c'est-à-dire sur des propositions données à l'avance. Toujours, en définitive, reproduire ainsi l'opinion établie, plutôt que de laisser place à l'inquiétude, que de laisser ouvert le lieu de la petite intranquillité tapie au sein de l'homme. Cri, question, ouverture, donc, face aux tautologies rassurantes et lénifiantes de l'opinion, et hélas, aussi trop souvent, de la philosophie.

Humeur existentielle : profonde lassitude, dégoût et ennui sécrétés par le monde constitué de part en part par Autrui, monde humain, trop humain. Plutôt faire le bête et l'ignorant que de participer à la tyrannie d'Autrui. Soyons de mauvaise volonté. Quand il prend la peine de ne pas considérer que tout va de soi, l'homme découvre en son sein une petite intranquillité, une insatisfaction fondamentale. Certains, trop las, voudront le rien, d'autres ne voudront plus rien. II en est tout autrement des deux auteurs qui ici nous occupent. Au-delà du penchant critique de leur œuvre quelque chose est pointé, quelque chose est âprement recherché. C'est ce que nous appelons l'innommable, hommage à l'un des plus grands chefs d'œuvre de Beckett. Voyons donc, parlons donc de ce qu'on ne peut pas dire, de cette expérience souhaitée, visée, désirée, se déprendre du monde pour Beckett, se fondre au monde pour Deleuze.

\section{Innommable. Se déprendre du monde, se fondre au monde}

On fait trop souvent, trop facilement de Beckett un nihiliste 29. C'est qu'on va trop vite, qu'on ne prête pas suffisamment attention à ce petit rien qui s'acharne une fois la trop solide et trop évidente jactance d'Autrui tue. Ni nihilisme, ni glorification de la vie, l'œuvre de Beckett trace une fine et subtile ligne entre la vie et la mort, entre les mots et le silence. Une fois le sujet aboli, l'enchaînement logique et évident d'Autrui troué et lacéré, reste un murmure qui s'acharne, murmure pour une conscience. Ce murmure qui vacille, sans certitudes, là où il pourrait ne pas être, mais ça s'acharne, ce murmure est composé de voix qui résonnent dans la conscience. «Elle (la voix) sort de moi, elle me remplit, elle clame contre mes murs, elle n'est pas la mienne, je ne peux pas l'arrêter, je ne peux pas l'empêcher de me déchirer, de me secouer, de m'assiéger»30. Mystère de la conscience. Elle est une sorte de chambre à écho où résonnent les voix, plus ou moins fortement. Elle est puissance et 
passion d'écoute, rappelons que pour Molloy, réfléchir c'est écouter plus fort. Mais cette écoute comme résonance ne doit pas donner l'illusion d'un dedans qui serait preuve de l'intériorité de la conscience. Comme le remarque bien Ludovic Janvier, si le narrateur de Comment c'est affirme "plus de voix au monde que la mienne" pour quelques pages plus tard dire au contraire "on parle de moi», c'est bien la preuve de «l'effort que fait la diction pour s'intérioriser : mais c'est en vain» ${ }^{31}$. La conscience est ainsi un non-lieu sans intériorité où une énigmatique présence est dissoute, portée, bercée, différée par des bribes, des blocs et des poussières de discours aliénateurs, puissance et passion d'écoute comme jaillissement promptement enseveli sous les mots qui résonnent en elle, par les mots qu'elle profère. Être vue et se dire sont deux passions fondamentales de la conscience. Être vue et se dire dans un procès continu rendant impossible toute présence à soi. Être vue, car la conscience en tant que conscience n'est telle qu'à condition d'être perçue. Ce qui signifie aussi être dite. Esse est percipi. Car la conscience a aussi ce drôle de sentiment d'être témoin d'elle-même, donc d'exister. Ce qui donnera le beau film de Beckett, Film, où le protagoniste essaie d'échapper à une perception, à un regard qui se trouve être le sien. Désir d'imperceptibilité. Mais la conscience tient, ce petit jaillissement différé s'acharne.

Une fois tue la clameur assourdissante de l'action, du dit d'Autrui, le filet, le murmure spiroïdal de la conscience prend son envol. Voyage immobile. Tendre à éliminer le plus de déterminations possibles - se déprendre du monde, se déprendre d'Autrui, pour se laisser bercer par le clapotis de voix, toujours des déterminations, mais moins massives, clapotis qui peut ainsi mieux se faire entendre. Entendre, faire résonner le monde des voix. Monde bigarré où clapir les voix du corps et les voix de l'enchaînement d'Autrui autrefois cohérent, se réduisant maintenant en bribes s'écoulant au hasard de leur procès, sans buts, sans origines, sans identités. Mots morts. Le corps pour Beckett est un donné qui insiste, donné encombrant et étranger à la conscience, «corps-objet, corps-boulet, corps-pourriture, corps-excrément»32. II émettra ses souffles, ses sons, il se parlera aussi, dans l'écoulement du dire, ajoutant ses voix à celles, naguère si fières, maintenant bribes incohérentes, du discours d'Autrui. Une fois délitée la fiction du sujet, il ne reste que l'énigmatique conscience et l'acharnement en son sein, en elle, par elle, de l'écoulement du dire. Ou plutôt de l'écho des voix et des mots. 
La mémoire aura aussi son rôle, mais comme le reste elle perd sa belle évidence et ne vient se faire entendre que par intermittence à la conscience, au gré des réminiscences involontaires.

Se déprendre du monde, se retrouver conscience qui elle même cherche à se dire, occultée par son dire, donc inquiète même dans cette non-présence à elle même, nous croyons que Beckett a posé d'une manière particulièrement aiguë le problème de l'existence. Car si l'existence fait problème, n'est-ce pas qu'elle est conscience problématique d'exister? Et que cette conscience voudrait tellement exister d'elle-même? Ou se dissoudre en un pur filet vagabond? Sortir d'elle-même? Pourquoi? Comment le pourrait-elle, d'ailleurs, elle qui n'est que pure surface?

\author{
«Être à l'heure du monde. Voilà le lien entre imperceptible, \\ indiscernable, impersonnel, les trois vertus. Se réduire à \\ une ligne abstraite (...)" \\ Deleuze et Guattari, Mille Plateaux, p.343
}

Être rafale, être cavale, le nomadisme comme ethos est le fin mot de l'existence selon Deleuze. Dans la société comme dans l'inconscient, dans tout individu, c'est la ligne de fuite qui est primordiale. Mais voilà, celle-ci est refoulée, bloquée, canalisée par le système de la représentation. L'ethos nomade est la prise en charge, la fuite grâce et pour la ligne de fuite. C'est une conjonction sans médiation avec le processus, c'est être le procès. Toujours cette idée de sortir de son moi étriqué, de ce sujet imposé du dehors, petite pièce parmi tant d'autres de l'infernale machinerie des obligations sociales. Profonde lassitude envers ce sempiternel jeu de rôle. Une des figures du refus existentiel absolu de ces rôles sera le schizophrène, non celui qu'on retrouve comme loque dans les hôpitaux parce qu'on a brisé son processus, mais celui qui traverse le mur. Mystère de la folie. Mais attention, ici aucune complaisance pour le "fou», héros romantique, contestataire, génial, figure stéréotypée d'un ordre social se mirant dans son envers. Le schizophrène pointe plutôt vers la nature de l'inconscient, libre, sans origine, ni but, processus de production, passion de couplage, de connexion. Pour Deleuze, c'est cet aspect de l'existence qu'il faut privilégier, vrai mouvement de la vie, tandis que le sujet, bien que nécessaire n'est qu'un reste, qu'un résultat que produit le processus. Le sujet n'est pas un je pense, mais un je contracte, un je sens. "C'est un étrange sujet, 
sans identité fixe, errant sur le corps sans organes, toujours à côté des machines désirantes, défini par la part qu'il prend au produit, recueillant partout la prime d'un devenir ou d'un avatar, naissant des états qu'il consomme et renaissant à chaque état $\$ 33$. Le sujet n'est qu'un résultat, un je deviens dans telle ou telle intensité présente. "Je est une habitude»34 dira Deleuze en citant Hume et les anglo-saxons, je est contemplation, le sujet est toujours issu d'une synthèse passive ${ }^{35}$, point de vue qui éclot et meurt pour laisser place à une autre habitude et ainsi de suite. Le je est une multiplicité de petits sujets apparaissant chaque fois qu'il y a habitude, affect, consommation, plaisir. On définit mieux le sujet par l'avoir que par l'être. Précision par contre que le sujet n'a pas des habitudes et des affects, mais que des habitudes et des affects créent d'éphémères états de sujet (le sujet comme j'ai eu). Mais voilà, cette multitude de sujets de préhension, tous ces états affectifs sont subsumés par un sujet réifié et tout puissant, rôle social correspondant à un système de production, petite histoire intime et personnelle sous-tendue par la psychanalyse ou la religion, nécessaire au système moral de la responsabilité. Une série d'effets, de restes du processus se réifie en une illusoire cause : Je. Alors se rigidifie l'habitude identitaire, refoulant le désir et ses divers états intensifs, certitudes et névroses du je de la représentation : intransigeance du je suis.

"Nous avons retenu d'CEdipe le sale petit secret, et non pas CEdipe à colonne, sur sa ligne de fuite, devenu imperceptible, identique au grand secret vivant»36. En finir avec le petit sujet névrosé constitué par le complexe d'CEdipe, affirmer la suffisance et la plénitude du désir, n'a jamais signifié pour Deleuze un abandon à la jouissance s'extrayant à tout prix de toute souffrance. Comme l'a si bien formulé Mengue ${ }^{37}$, le désir chez Deleuze est errance de l'illimité, véritable souffrance de l'existant face au 'grand secret vivant' et non petites souffrances organisées, insufflées, distillées au sujet par les tripatouilleurs de l'âme que sont les curés et les psychanalystes. Le désir est joie et passion de l'existant pourfendu par cette errance de l'illimité que toute société tente de circonscrire, de bloquer, de refouler. Acharnement à rompre les amarres, le désir donne l'existant à cette errance de l'illimité, sans but, sans fin, sans origine. Cri et béance problématique comme promesse de cette errance. Devenir-imperceptible car cette errance rend étranger à la communauté de ses semblables celui qui file au-delà de la structure Autrui. C'est-à-dire déliter le sujet-habitude de la représentation, socle 
identitaire réifié, pour laisser l'existant explorer ses propres champs intensifs, se laisser aller à un devenir-métamorphose incessant. Devenir-ligne dira aussi Deleuze, énigmatique errance qui en définitive doit tendre à se confondre avec le monde, filer avec le monde en le faisant filer en retour, fuser avec le monde. Se faire ligne pour mieux se tisser avec le monde : passion de la conjonction. Alors se confondre avec le mystère de la vie ${ }^{38}$, pure ligne, mystère de ces lignes selon Deleuze qui tissent le monde et qui débordent le vivant de toute part. Et, ligne, qui dans le vivant homme, s'acharne, laboure le creux de son existence, le poussant toujours au-delà de lui-même, errance de l'illimité dans une forme finie, mais il y a la pensée, l'énigme de la conscience, le désir, la vie, la mort. Errance de l'illimité.

Pris dans les rets d'Autrui, je suis sujet malgré moi, me pensant moi vociférant ce que je crois sortir de moi. Beckett et Deleuze, chacun à leur manière, expriment une extrême lassitude envers le monde d'Autrui et ses tranquilles évidences. Beckett met l'accent sur tout ce qui est charrié par nous, malgré nous par le langage, affres du monde de l'habitude, tandis que Deleuze débusque la structure Autrui source de notre confort mondain. Ce qu'on reproche à ce monde constitué de part en part par Autrui, même seul j'entends et je vois la cacophonie des autres, c'est d'anesthésier une souffrance en l'homme, véritable intensité d'une vie qui laboure l'homme de son profond sillon. Mais attention, ici, il ne s'agit plus d'attribuer souffrances à un sujet authentique et romantique. Plates complaintes recyclées, délavées, scories de tout ce qui n'a jamais été cru ou dit, baume d'oubli pour mieux se fondre dans la masse de ses semblables. Comme le suggère Lacan, l'angoisse est cette protection faisant sujet placée avant et masquant le fait que du sujet, il n'y a de place nulle part. Désarroi absolu qu'atteignent selon nous nos deux auteurs, acharnement du désir chez Deleuze, errance de l'illimité qui me donne, joie et souffrance, pleinement au monde, acharnement de cette petite lueur de la conscience chez Beckett, paradoxale conscience qui croit être particulière, sans cesse recouverte par ce qui la déborde, qui survole ce qui la déborde et la constitue, énigmatique présence d'une non-présence.

L'existence, comme fait le plus intime, a longtemps, trop longtemps été l'attribut privilégié d'un sujet, qu'on peut maintenant soupçonner de complaisance envers sa propre souffrance : l'existentialisme comme romantisme et héroïsme du sujet souffrant. L'angoisse en ce sens n'est pas ce qui menace le sujet, mais ce qui le 
conforte : créditons donc Beckett et Deleuze d'avoir exploré un nouvel espace existentiel plus fondamental, au-delà de la forme-sujet, désarroi absolu, "souffrance d'être» et "état provisoire de lucidité aiguë» selon le premier, errance de l'illimité selon le second. Étranges avatars d'un existentialisme pour un sujet dissous, passion d'errance qui pointe vers une énigmatique communion avec le monde, ou vers une non moins énigmatique conscience sans monde, témoin contemplatif victime de logorrhée. Acharnement à laisser la plaie du rapport existant-monde bien ouverte, entretenir sa béance et sa vivacité.

\section{Vincent Jacques}

\section{Bibliographie}

Beckett, S., Molloy, Paris, Éditions de Minuit, 1951

Beckett S., Malone meurt, Paris, Éditions de Minuit, 1951

Beckett, S., Innommable, Paris, Éditions de Minuit, 1953

Beckett, S., En attenant Godot, Paris, Éditions de Minuit, 1952

Beckett, S., Le monde et le pantalon, Paris, Éditions de Minuit, 1990

Beckett, S., Proust, Paris, Éditions de Minuits, 1990

Beckett, S., Trois dialogues, Paris, Éditions de Minuit, 1998

Deleuze G., Différence et répétition, Paris, PUF, 1968

Deleuze G., Logique du sens, Paris, Éditions de Minuit, 1969

Deleuze et Guattari F., l'Anti-CEdipe, Paris, Éditions de Minuit, 1972

Deleuze G. , Dialogue, Paris, Éditions de Minuit, 1977

Deleuze G. et Guattari F., Mille plateaux, Paris, Éditions de Minuit, 1980

Deleuze, G., Critique et clinique, Paris, Éditions de Minuit, 1993

Gauer, D., Le discours de la première personne dans les textes en prose de Samuel Beckett, thèse inédite, Université Lille III, 1996

Heidegger, M., Acheminement vers la parole, Paris, Gallimard, 1976

Janvier, L., Pour Samuel Beckett, Paris, Éditions de Minuits, 1966

Lacan, J., L'éthique de la psychanalyse (le séminaire volume VII), Paris, Le Seuil, 1986

Mengue, P.,Gilles Deleuze ou le système du multiple, Paris, Éditions Kimé, 1994

Rousset, B., Geulincx entre Descartes et Spinoza, Paris, Vrin, 1999

Sartre, J.P, L'être et le néant, Paris, Gallimard, 1943 
1. Innommable, référence à l'œuvre de Beckett du même nom.

2. Lacan, grand lecteur de Kierkegaard, affirme en effet que la cure psychanalytique réussie doit atteindre à l'expérience d'un "désarroi absolu, au niveau duquel l'angoisse est déjà une protection». L'éthique de la psychanalyse, p.351.

3. Le monde et le pantalon, p.35

4. L'innommable, p.18

5. Molloy, p. 150

6. Proust cité par Beckett in Proust, p. 38

7. Ibid., p.40

8. Geulincx, cité par Rousset dans Geulincx entre Descartes et Spinoza, p.49. (Nous traduisons). Notons combien ce livre est précieux, jetant un peu de lumière sur ce philosophe oublié de l'histoire de la philosophie.

9. Gauer, Le discours de la première personne dans les textes en prose de Samuel Beckett, thèse inédite, Lille III

10. Trois dialogues, p.14

11. Logique du sens, p.360

12. Ibid. p.356

13. Différence et répétition, p. 360

14. Logique du sens, p.370

15. Différence et répétition, p.94

16. Logique du sens, p.361

17. Mille Plateaux, p.95

18. Ibid., p.100

19. L'innommable, p.18

20. Innommable, p.163

21. Ibid., p.17

22. Proust, p.40

23. L'art de ne pas lire, p.9

24. «L'homme parle seulement pour autant qu'il répond au langage en écoutant ce qu'il lui dit» in Acheminement vers la parole, p. 236

25. "The key word in my plays is perhaps" (nous traduisons) cité par Gauer, op. cit., p.214

26. Différence et répétition, p.171.

27. Qu'est-ce que la philosophie, p.11

28. Dialogue, p.7

29. «Mais je n'étais pas fait pour la grande lumière qui annihile, on ne m'avait donné qu'une petite lampe et une grande patience, pour me promener dans les ombres vides" Molloy, p.179

30. Innommable, p.34

31. Pour Samuel Beckett, p.209

32. Gauer, op.cit., p.185

33. Anti-CEdipe, p.23

34. Qu'est-ce que la philosophie, p.101 


\section{Vincent Jacques}

35. Synthèse passive, car celle-ci se produit sans l'aide de la réflexion. En contractant les forces dont il procède un sujet se crée. La synthèse est ce qui est vécu dans un affect, préhension physiologique ou psychologique. L'important est que le sentir, la contraction, s'attribue, se contemple, je sens, je contracte, créant ainsi un sujet (C'est à moi, c'est moi). Tous ces micro-sujets, ce clapotement de petites synthèses, sont le lit sur lequel le Je réifié se båtit, habitudes sociales, habitude de dire je, synthèses actives du sujet à l'identité fixe et stable de la représentation.

36. Dialogue, p.58

37. Gilles Deleuze ou le système du multiple, p.165

38. “Devenir imperceptible est la Vie, 'sans cesse ni condition', atteindre au clapotement cosmique et spirituel', 